\title{
Nitrotyrosine and 8-isoprostane formation indicate free radical-mediated injury in hearts of patients subjected to cardioplegia
}

Uwe Mehlhorn, MD, ${ }^{a}$ Andreas Krahwinkel, PhD, ${ }^{\mathrm{b}}$ Hans J. Geissler, MD, ${ }^{\mathrm{a}}$ Karl LaRosee, MD, ${ }^{\mathrm{c}}$ Uwe M. Fischer, MD, ${ }^{\mathrm{a}}$ Oliver Klass, MD, ${ }^{a}$ Michael Suedkamp, MD, ${ }^{a}$ Khrosro Hekmat, MD, ${ }^{a}$ Paschalis Tossios, MD, a and Wilhelm Bloch, MD ${ }^{\mathrm{b}}$

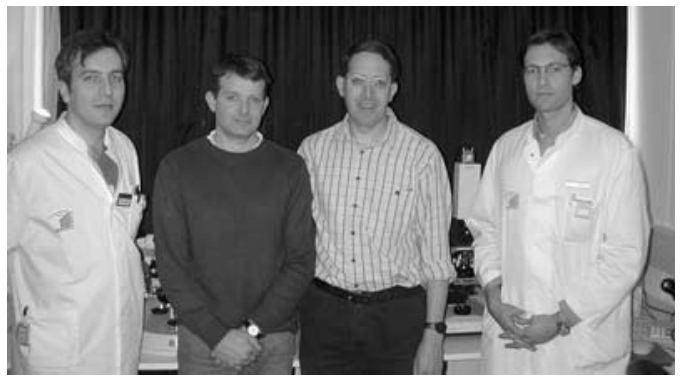

Tossios, Bloch, Krahwinkel, Mehlhorn (left to right)

Objective: Myocardial ischemia and reperfusion induced by cardioplegic arrest subjects the heart to free radical-mediated stress. The purpose of our study was to investigate the effect of cardioplegia-induced ischemia and reperfusion on myocardial formation and distribution of (1) nitrotyrosine as an indicator for peroxynitrite-mediated tissue injury resulting from increased nitric oxide release and (2) 8-isoprostane as an indicator for oxygen-derived free radical-mediated lipid peroxidation.

Methods: In 10 patients undergoing coronary artery operations (64 \pm 6 [mean $\pm \mathrm{SD}$ ] years, 3 women and 7 men) subjected to cardiopulmonary bypass and intermittent cold blood cardioplegia, we collected transmural left ventricular biopsy specimens before and at the end of cardiopulmonary bypass. Specimens were cut at $10 \mu \mathrm{m}$ and subjected to immunocytochemical staining against the nitric oxide-producing enzyme constitutive nitric oxide synthase, cyclic guanosine monophosphate (intracellular second messenger of nitric oxide), nitrotyrosine, and 8-isoprostane by using polyclonal antibodies. For global left ventricular function determination, we measured the fractional area of contraction using transesophageal echocardiography.

From the Departments of Cardiothoracic Surgery, ${ }^{\mathrm{a}}$ Anatomy, ${ }^{\mathrm{b}}$ and Cardiology, ${ }^{\mathrm{c}}$ University of Cologne, Cologne, Germany.

Received for publication Nov 27, 2001; revisions requested Feb 18, 2002; revisions received Feb 25, 2002; accepted for publication April 8, 2002.

Address for reprints: Uwe Mehlhorn, MD, Professor, Department of Cardiothoracic Surgery, University of Cologne, JosephStelzmann-Str 9, 50924 Cologne, Germany (E-mail: uwe.mehlhorn@medizin.unikoeln.de).

J Thorac Cardiovasc Surg 2003;125:178-83

Copyright $(\odot 2003$ by The American Association for Thoracic Surgery

$0022-5223 / 2003 \$ 30.00+0$

doi:10.1067/mtc. 2003.97
Results: Nitric oxide synthase activity in cardiac myocytes increased from $34 \pm 10$ gray units before cardiopulmonary bypass to $47 \pm 12$ gray units at the end of bypass $(P=$ $.015)$, and all hearts showed increased cyclic guanosine monophosphate content in both myocytes and endothelial cells at the end of bypass. The number of nitrotyrosinepositive capillaries increased from $36 \pm 29 / \mathrm{mm}^{2}$ before bypass to $82 \pm 47 / \mathrm{mm}^{2}$ at the end of bypass $(P=.012)$, and 8-isoprostane-positive capillaries increased from $92 \pm$ $72 / \mathrm{mm}^{2}$ before bypass to $209 \pm 108 / \mathrm{mm}^{2}$ at the end of bypass $(P=.005)$. The fractional area of contraction was $53 \% \pm 12 \%$ before bypass and $56 \% \pm 12 \%$ after bypass $(P=.47)$ but was slightly decreased to $45 \% \pm 14 \%$ at 4 hours after bypass $(P=.121)$.

Conclusions: Our data show that cardioplegia-induced myocardial ischemia and reperfusion is associated with nitrotyrosine and 8-isoprostane formation mainly in the coronary endothelium, indicating injury mediated by both peroxynitrite and oxygen-derived free radicals. Because nitric oxide synthase activation was accompanied with increased cyclic guanosine monophosphate, these data suggest that direct effects of nitric oxide on cardiac myocytes, as well as nitric oxide-mediated coronary endothelial injury, might contribute to injury associated with cardioplegia and cardiopulmonary bypass.

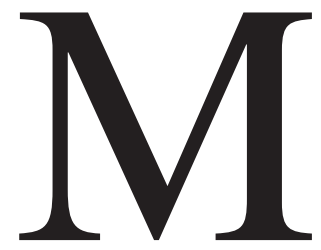

yocardial ischemia and reperfusion subjects the heart to stress mediated by free oxygen radicals or reactive oxygen species (ROS), such as hydroxyl radical $\left(\mathrm{OH}^{-}\right)$, superoxide radical $\left(\mathrm{O}_{2}{ }^{-}\right)$, hydrogen peroxide $\left(\mathrm{H}_{2} \mathrm{O}_{2}\right)$, and peroxynitrite $\left(\mathrm{NO}_{3}{ }^{-}\right)$, the product of nitric oxide (NO) binding to $\mathrm{O}_{2}{ }^{-} \cdot{ }^{1-3} \mathrm{ROS}$ are thought to mediate the reperfusion injury that characterizes metabolic, structural, and functional (stunning) myocardial damage associated with 
experimental coronary occlusion and reperfusion. ${ }^{1-3}$ However, little evidence of such a relationship exists in human subjects. This is in part due to the limitations intrinsic to available indices of ROS-mediated tissue injury in vivo. ${ }^{4}$ Thus clinical investigators have mainly relied on ex vivo spin-trapping techniques and determination of indirect evidence for ROS-mediated lipid peroxidation, such as malondialdehyde and lipid hydroxyperoxydase determination, in the blood of patients with acute myocardial infarction ${ }^{5}$ or those subjected to percutaneous transluminal coronary angioplasty $^{6}$ or coronary artery bypass grafting $(\mathrm{CABG}){ }^{7}$ More recently, a number of novel assays of more stable ROS-mediated tissue injury markers, such as 8 -iso-prostaglandin- $\mathrm{F}_{2} \alpha$ (8-iosprostane) $)^{1,8}$ and nitrotyrosine ${ }^{9,10}$ have been described. 8-Isoprostane is the stable end product of arachidonic acid oxidation generated by ROS attacks on membrane phospholipids. ${ }^{11}$ Nitrotyrosine represents the stable end product of cell membrane protein-bound tyrosine nitration by peroxynitrite caused by increased NO. ${ }^{9}$

We have recently shown, in patients undergoing CABG, that cardioplegia-induced myocardial ischemia and reperfusion activates the NO-producing enzyme constitutive NO synthase (constitutive NOS or NOS-III) in cardiac myocytes and endothelium, leading to increased NO release. ${ }^{12}$ In addition, Hayashi and colleagues ${ }^{13}$ demonstrated that human myocardium produces peroxynitrite during reperfusion after cardioplegic arrest. These data suggest that peroxynitrite-mediated nitrotyrosine formation caused by NOS-III activation and NO release might contribute to ROS-induced reperfusion injury after cardioplegic arrest. However, direct evidence for cardioplegia-induced reperfusion injury by ROS is still lacking. Therefore the purpose of our study was to investigate the effect of cardioplegia-induced ischemia and reperfusion on myocardial formation and distribution of nitrotyrosine as an indicator for peroxynitrite-mediated tissue injury and 8-isoprostane as an indicator for ROS-mediated lipid peroxidation.

\section{Material and Methods \\ Patients}

After obtaining approval from the University of Cologne Human Ethics Committee, written informed consent was obtained from each patient during the preoperative interview. Ten patients scheduled for elective coronary artery surgery (Table 1 ) were included in the study. Patients were subjected to cardiopulmonary bypass (CPB) at $32^{\circ} \mathrm{C}$ to $34^{\circ} \mathrm{C}$, the aorta was crossclamped, and intermittent (every 20 minutes) cold $\left(6^{\circ} \mathrm{C}-8^{\circ} \mathrm{C}\right)$ antegrade blood cardioplegia with hot shot before crossclamp removal (according to the method of Buckberg) was administered.

\section{Clinical Protocol}

After anesthesia induction and standard hemodynamic monitoring, a 5-MHz transesophageal echocardiography probe (Vingmed CFM $800^{\circ}$; Sonotron, Horten, Norway) was placed to provide a left ventricular (LV) short-axis image at the midpapillary level. From
TABLE 1. Patient data

\begin{tabular}{lc}
\hline Patients (n) & 10 \\
Female/male (n) & $3 / 7$ \\
Age (y) & $63.7 \pm 6.3$ \\
Height (cm) & $168.7 \pm 7.0$ \\
Weight (kg) & $78.8 \pm 9.7$ \\
1/2/3-Vessel disease (n) & $0 / 1 / 9$ \\
Angiographic LV ejection fraction (\%) & $64.4 \pm 11.7$ \\
LVEDP (mm Hg) & $13.3 \pm 8.3$ \\
Grafts per patient (n) & $3.5 \pm 0.5$ \\
LITA to LAD (n) & $1.0 \pm 0.0$ \\
CPB duration (min) & $95.8 \pm 14.9$ \\
AXC duration (min) & $56.5 \pm 10.1$ \\
Postoperative CK-MB ${ }_{\text {max }}$ (U/L) & $12.9 \pm 4.8$ \\
Perioperative myocardial infarction (n) & 0
\end{tabular}

LVEDP, Left ventricular end-diastolic pressure; LITA, left internal thoracic artery; $L A D$, left anterior descending artery; $A X C$, aortic crossclamp; $C K$ $M B$, creatine kinase MB fraction. Data are given as mean $\pm \mathrm{SD}$.

the transesophageal echocardiography recordings, we derived the fractional area of contraction (FAC) as a measure of LV ejection fraction before cannulation for $\mathrm{CPB}$, at 10 to 15 minutes after weaning from $\mathrm{CPB}$, and at 4 hours after $\mathrm{CPB}$ in the intensive care unit. ${ }^{14}$

\section{Biopsy Specimens}

Before CPB initiation, we collected a transmural biopsy specimen from a fat-free area of the LV anterior wall using a 14-gauge biopsy needle (Gallini, Modena, Italy). A second LV biopsy specimen was taken at the end of the extracorporeal circulation before weaning from CPB. All LV biopsy specimens were placed in $4 \%$ paraformaldehyde for 4 hours and then rinsed in $0.1 \mathrm{~mol} / \mathrm{L}$ phosphate-buffered saline (PBS) solution for 24 hours, followed by storage for 12 hours in PBS solution with $18 \%$ sucrose for cryoprotection, and frozen at $-80^{\circ} \mathrm{C}$. Because $4 \%$ paraformaldehyde has been shown to result in optimal fixation of cyclic guanosine monophosphate (cGMP) on the cell's protein matrix, ${ }^{15}$ we used this solution for immunohistochemical detection of the watersoluble cGMP.

\section{Immunocytochemistry}

Before immunohistochemical examination, 10- $\mu \mathrm{m}$ slices from the biopsy specimens were placed in a bathing solution of $3 \% \mathrm{H}_{2} \mathrm{O}_{2}$ and $60 \%$ methanol PBS for 30 minutes and then permeabilized with $0.2 \%$ Triton-X 100 in $0.1 \mathrm{~mol} / \mathrm{L}$ PBS. Thereafter, specimens were treated with $5 \%$ normal goat serum and $5 \%$ bovine serum albumin solution in PBS. Before each step, the sections were rinsed 3 times in PBS buffer. Incubation with primary polyclonal rabbit anti-NOS-III antibody (Biomol, Hamburg, Germany) at a dilution of 1:1500 was performed in a PBS-based solution of $0.8 \%$ bovine serum albumin and $20 \mathrm{mmol} / \mathrm{L} \mathrm{NaN}_{3}$ for 12 hours at $4{ }^{\circ} \mathrm{C}$. For cGMP detection, we used a polyclonal rabbit anti-cGMP antibody (Quartett, Hamburg, Germany) at a dilution of 1:600. After rinsing with PBS, the sections were incubated with the corresponding secondary biotinylated goat anti-rabbit antibody (Vector Laboratories, Burlingame, Calif) for 1 hour at room temperature. For nitrotyrosine staining, we used a monoclonal mouse anti-nitrotyrosine antibody (Calbiochem, San Diego, Calif) at 
NOS-III
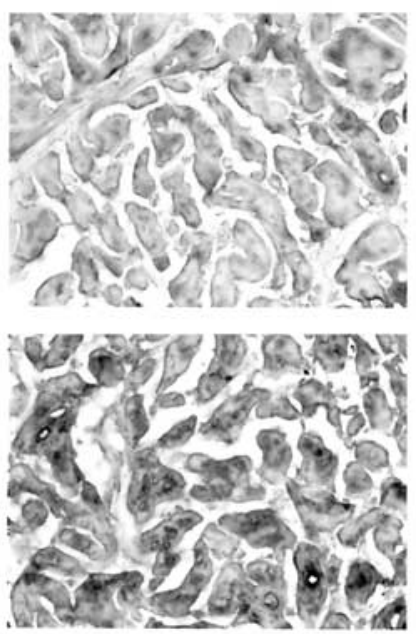

cGMP
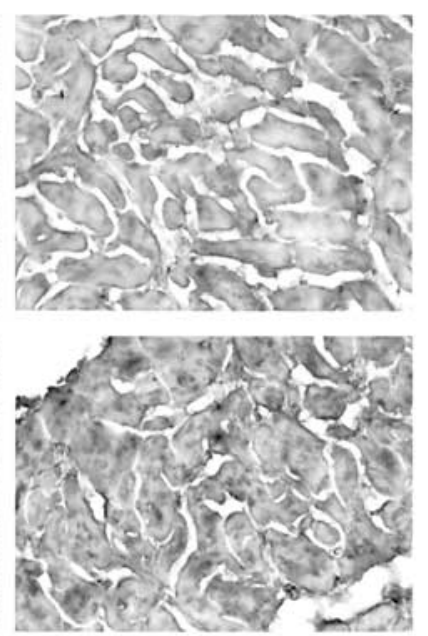

nitrotyrosine
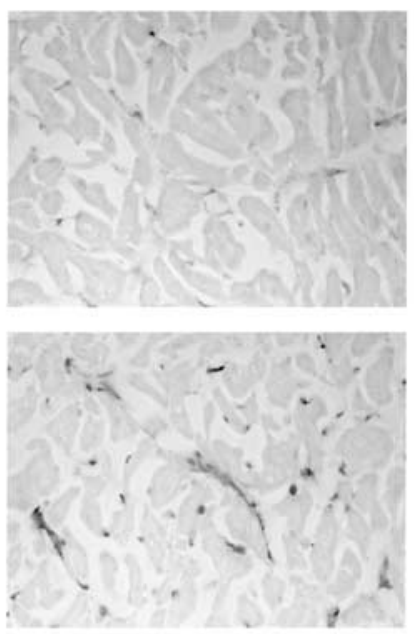

\section{8-isoprostane}
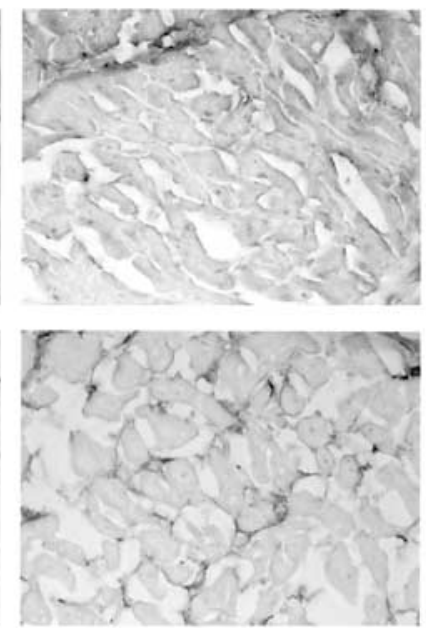

Figure 1. NOS-III activity and cGMP content were increased in both cardiac myocytes and endothelial cells at the end of CPB (lower image line) compared with before CPB (upper image line). Nitrotyrosine and 8-isoprostane activity increased mainly in coronary endothelial cells.

1:400 and a secondary goat anti-mouse antibody (DAKO, Weisloch, Germany) at 1:400; for 8-isoprostane detection, a polyclonal goat anti-8-Epi-PGF2a antibody (Oxford Biomedical Research, Oxford, Mich) at 1:1500 and a secondary rabbit anti-goat antibody (DAKO, Germany) at 1:400 was used. A streptavidin-horseradish peroxidase complex was then applied as a detection system (1:100 dilution) for 1 hour. Finally, staining was developed for 3 to 5 minutes with 3,3-diaminobenzidine tetrahydrochloride in 0.05 $\mathrm{mol} / \mathrm{L}$ TRIS-HCl buffer and $0.1 \% \mathrm{H}_{2} \mathrm{O}_{2}$.

\section{NOS-III Television Densitometry}

All LV biopsy slices were incubated and stored under identical conditions. For intensity analysis of NOS-III immunostaining in cardiomyocytes, we measured the gray values of 50 cardiomyocytes from 10 randomly selected areas. The intensity of immunostaining was reported as the mean of measured cardiomyocyte gray value minus background gray value. The background gray value was measured at a cell-free area of the slice. For staining intensity detection, a Zeiss Axiophot microscope coupled to a 3-chip CCD camera was used, and the analysis was performed by using the Optimas 6.01 image analysis program installed on a Pentium personal computer.

\section{cGMP Semiquantitative Analysis}

For semiquantitative analysis of myocardial cGMP content, we used a score to differentiate between no change and clearly increased cGMP content. All specimens were independently judged by 2 investigators in a blind fashion. Any disagreement between judgements was subsequently resolved by discussion.

\section{Nitrotyrosine and 8-Isoprostane Quantification}

Quantitative analysis was performed on 5 randomly selected fields $\left(66,125 \mu \mathrm{m}^{2}\right.$ per frame) of LV biopsy cross-sections. All immu- nohistochemically stained capillaries per field were counted with a Zeiss Axiophot microscope with a $40 \times$ oil immersion objective and expressed as number per square millimeter.

\section{Statistical Analysis}

All data are presented as mean \pm SD. Data were analyzed for statistical significance on an $\alpha$ levels of $5 \%$ by using the 2-tailed Student $t$ test for paired samples, as implemented in the software package SASS for Windows, version 10.0. The $P$ values reported are not adjusted for multiple testing.

\section{Results}

Myocardial immunostaining for NOS-III, cGMP, nitrotyrosine, and 8-isoprostane is depicted in Figure 1. Compared with before CPB (Figure 1, upper line), NOS-III activity and cGMP content were increased in both cardiac myocytes and endothelial cells at the end of CPB (Figure 1, lower line). Nitrotyrosine and 8-isoprostane activity increased mainly in myocardial capillaries (Figure 1).

Because the small size of the LV biopsy samples, we were able to determine NOS-III activity and cGMP content in 9 hearts (before $\mathrm{CPB}$ and at the end of $\mathrm{CPB}$ ), nitrotyrosine-positive capillaries in 6 hearts, and 8-isoprostanepositive capillaries in 5 hearts. NOS-III activity in cardiac myocytes increased significantly from $34 \pm 10$ gray units (U) before CPB to $47 \pm 12 \mathrm{U}$ at the end of CPB $(P=.015$, Figure 2), and all hearts showed increased cGMP content in both myocytes and endothelial cells. The number of nitrotyrosine-positive capillaries increased significantly from $36 \pm 29 / \mathrm{mm}^{2}$ before CPB to $82 \pm 47 / \mathrm{mm}^{2}$ at the end of CPB $(P=.012$, Figure 3$)$, and the number of 8 -isoprostane- 


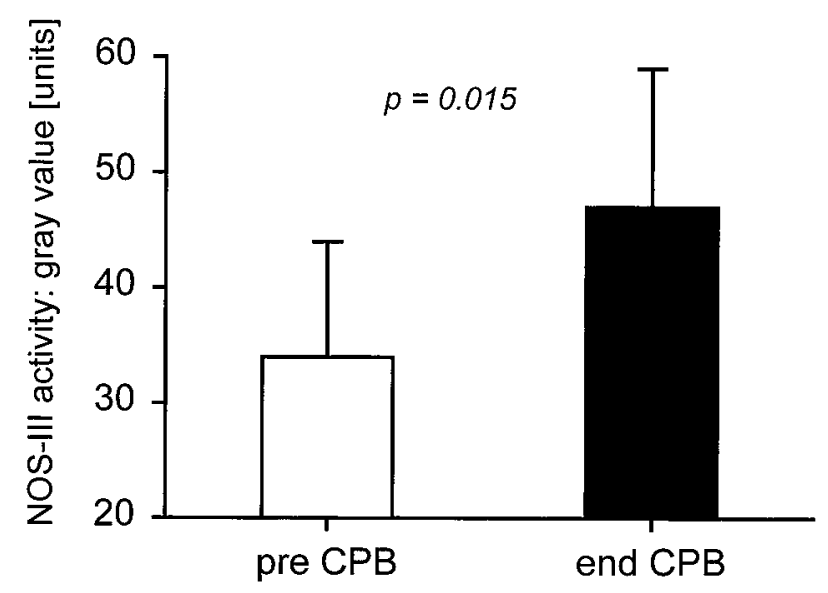

Figure 2. At the end of CPB, NOS-III activity, as determined by using television densitometry, was increased compared with that before CPB. Bars represent mean values, with whiskers indicating corresponding SDs $(\mathrm{n}=9)$.

positive capillaries increased significantly from $92 \pm 72 /$ $\mathrm{mm}^{2}$ before CPB to $209 \pm 108 / \mathrm{mm}^{2}$ at the end of CPB $(P=$ .005 , Figure 4$)$. There was no correlation between the increases in NOS-III, nitrotyrosine-positive capillaries, or 8 -isoprostane-positive capillaries versus $\mathrm{CPB}$ duration or aortic crossclamp time, respectively $\left(r^{2}<0.35\right)$. Global LV ejection fraction, as assessed by means of FAC, was $53 \% \pm$ $12 \%$ before CPB and $56 \% \pm 12 \%$ after CPB $(P=.47)$ but was slightly decreased to $45 \% \pm 14 \%$ at 4 hours after CPB $(P=.121)$.

\section{Discussion}

Our data show that cardioplegia-induced myocardial ischemia and reperfusion is associated with nitrotyrosine and 8-isoprostane formation mainly in the coronary endothelium. Thus our study for the first time provides direct evidence for ROS-mediated tyrosine nitration and lipid peroxidation of myocardial endothelial membranes in patients subjected to cardioplegic arrest. Specifically, these data suggest that peroxynitrite production caused by NOS-III activation and increased NO release contributes to myocardial injury after cardioplegic arrest and CPB.

Ischemia-reperfusion injury involves both cardiac myocytes and coronary endothelial cells and appears to be a major factor contributing to perioperative myocardial damage. ${ }^{16}$ Although the pathophysiology of ischemia-reperfusion injury is not yet fully understood, recent studies suggest that ROS mediate the reperfusion injury that characterizes metabolic, structural, and functional myocardial damage associated with experimental coronary occlusion and reperfusion. ${ }^{1-3}$ Specifically, the role of NO and its ROS peroxynitrite $\left(\mathrm{NO}_{3}{ }^{-}\right)$has been studied extensively; however, the various studies have yielded conflicting data showing both

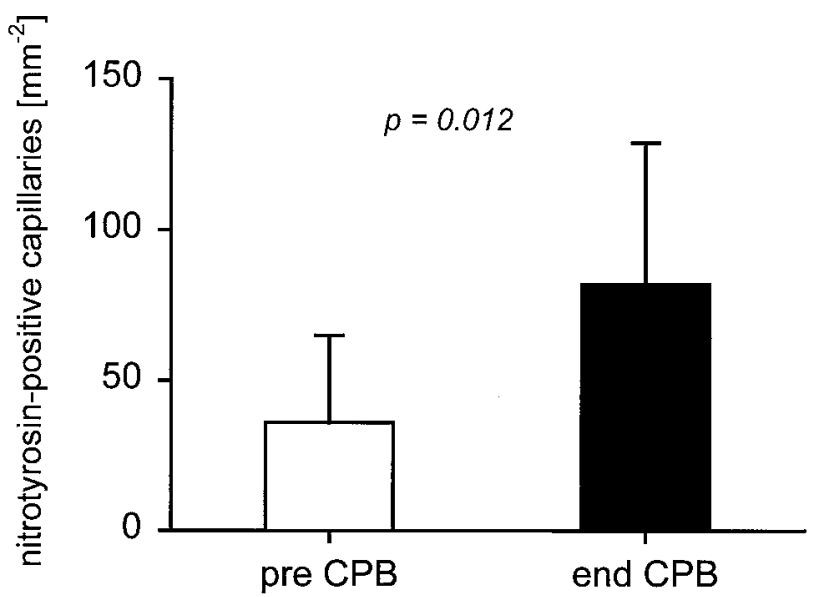

Figure 3. The number of nitrotyrosine-positive capillaries in human myocardium subjected to blood cardioplegia and reperfusion was increased at the end of CPB compared with before CPB, indicating injury induced by peroxynitrite, which was caused by increased NO release. Bars represent mean values, with whiskers indicating corresponding SDs $(n=6)$.

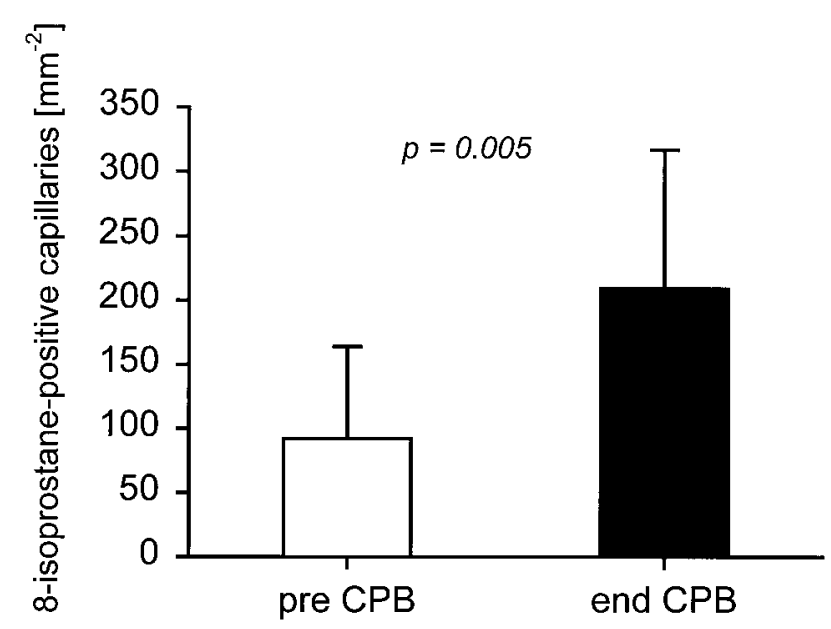

Figure 4. The number of 8-isoprostane-positive capillaries in human myocardium subjected to blood cardioplegia and reperfusion was increased at the end of CPB compared with before CPB, indicating oxygen-derived free radical-mediated lipid peroxidation. Bars represent mean values, with whiskers indicating corresponding SDs $(n=5)$.

protective, as well as deleterious, NO effects during reperfusion. Cardioprotective NO effects include reduction of neutrophil and platelet accumulation, inhibition of the release of cytotoxic mediators from neutrophils, direct cytoprotective effects on both endothelial cells and cardiomyocytes, ${ }^{16}$ and potential infarct size reduction. ${ }^{17}$ However, increased NO release has been suggested to contribute to reperfusion injury through peroxynitrite-mediated membrane nitration, DNA synthesis inhibition, ${ }^{18}$ mitochondrial 
function inhibition, ${ }^{19}$ ribonucleotide reductase inhibition, ${ }^{20}$ and cytokine-induced myocardial contractile dysfunction. ${ }^{21}$ These data suggest that high NO levels during reperfusion might be as deleterious as NO absence, and thus the role of $\mathrm{NO}$ in ischemia and reperfusion might be dose dependent.

Our and other groups' recent experimental work showed that myocardial ischemia acts as the stimulus for NOS-III activation, probably by inducing a conformational change of the enzyme, resulting in increased NO release as indicated by increased intracellular cGMP, NO's intracellular second messenger. ${ }^{22,23}$ Similarly, we have demonstrated, in patients undergoing $\mathrm{CABG}$, that cardioplegic arrest is associated with NOS-III activation and increased cGMP in both cardiac myocytes and endothelial cells, indicating increased NO formation. ${ }^{12}$ To further elucidate the pathophysiology of NO in ischemia and reperfusion, we intended to investigate whether the increased NO release caused by NOS-III activation results in myocardial tyrosine nitration as direct evidence for tissue injury. In the present study the number of nitrotyrosine-positive capillaries was significantly increased after cardioplegia (Figure 3), indicating peroxynitrite formation in the myocardium. This is in agreement with recent work by Hayashi and colleagues, ${ }^{13}$ who demonstrated transcoronary peroxynitrite production during reperfusion in patients subjected to cardioplegic arrest. These data suggest that peroxynitrite-mediated nitrotyrosine formation caused by NOS-III activation and increased NO release contributes to ROS-induced myocardial injury after cardioplegic arrest and CPB.

In addition to peroxynitrite, other ROS, including hydroxyl radical $\left(\mathrm{OH}^{-}\right)$, superoxide radical $\left(\mathrm{O}_{2}{ }^{-}\right)$, and hydrogen peroxide $\left(\mathrm{H}_{2} \mathrm{O}_{2}\right)$, have been suggested to be involved in ischemia-reperfusion injury. ${ }^{1-3}$ One novel marker for ROSmediated membrane lipid oxidation is 8-isoprostane, the stable end product of arachidonic acid oxidation. ${ }^{8,11}$ Investigators have determined 8-isoprostane as a marker of oxidative stress in urine ${ }^{1}$ or blood ${ }^{24}$ of patients subjected to percutaneous transluminal coronary angioplasty with acute myocardial infarction; however, myocardial 8-isoprostane formation and distribution caused by cardioplegia have not been studied. In the present study the number of 8-isoprostane-positive capillaries in human myocardium was significantly increased at the end of CPB (Figure 4). These data provide direct evidence for the contribution of ROS-mediated membrane lipid oxidation to ischemia-reperfusion injury in patients subjected to intermittent cold-blood cardioplegia. Interestingly, 8-isoprostane and nitrotyrosine formation were mainly confined to coronary endothelium, and only little staining was observed in cardiac myocytes (Figure 1). This is probably because of the high cardiac myocyte superoxide dismutase concentration, ${ }^{25}$ resulting in only little myocyte ROS, and thus 8-isoprostane, as well as nitrotyrosine, formation, even in the presence of increased $\mathrm{NO}$ as $\mathrm{NO}$, requires $\mathrm{O}_{2}{ }^{-}$to form $\mathrm{NO}_{3}{ }^{-} \cdot{ }^{16}$

\section{Functional Implications}

The functional correlate of ROS-mediated tissue injury is myocardial stunning. ${ }^{2,3}$ Recent experimental studies have shown that peroxynitrite production caused by increased NO release is associated with irreversible cardiac function impairment ${ }^{26}$ and higher mortality after coronary occlusion $^{27}$ and might be involved in cardiac apoptosis initiation. ${ }^{10}$ Gupte and Okada ${ }^{28}$ recently demonstrated that ROS mediate LV dysfunction through peroxynitrite production that can be attenuated by NOS inhibition. In addition, 8-isoprostane has been shown to induce coronary vasoconstriction associated with lactate production and contractile dysfunction. ${ }^{29}$ This coronary vasoconstriction persisted even after removal of the ROS stimulus and might thus contribute to the no-reflow phenomenon during reperfusion. ${ }^{30}$ In the present study global LV ejection fraction, as measured by means of FAC, was unchanged at 10 to 15 minutes after separation from CPB but was slightly decreased at 4 hours after CPB. Because these patients received continuous infusion of $3.9 \pm 1.0 \mu \mathrm{g} \cdot \mathrm{min}^{-1} \cdot \mathrm{kg}^{-1}$ dopamine after CPB, this mild LV dysfunction could reflect the functional ischemia-reperfusion injury associated with the structural LV myocardial changes we observed. However, other factors, including changed loading conditions or decreased endogenous catecholamines, could also have affected LV performance.

\section{Limitations of the Study}

Because we did not include a treatment group (ie, addition of a radical scavenger or NOS inhibitor) in our study, the present findings have to be regarded as preliminary observations. Furthermore, ROS are only one aspect in the mutifactorial process of ischemia-reperfusion injury, which involves several factors, such as physical, metabolic, and immunologic components. Thus the immunohistochemical changes we observed might not be causally related to injury directly caused by ischemia and reperfusion. Further studies will have to show that pharmacologic interventions can decrease the tissue content of ROS-induced changes and that this will be associated with improved cardiac performance to prove the clinical relevance of ROS. In addition, the data of the present study do not allow us to separate changes induced by CPB from those caused by cardioplegia and reperfusion. Thus CPB itself could have been the source of some or all ROS-mediated changes we observed. However, this is unlikely because we have previously shown that LV NOS-III and cGMP were not activated when using continuous antegrade coronary perfusion with normothermic CPB blood enriched with the $\beta$-blocker esmolol, a nonischemic myocardial protection method during aortic 
crossclamping. ${ }^{12}$ Together with the experimental data from our and other groups, ${ }^{22,23}$ these data suggest that ischemia acts as a stimulus for NOS-III activation. Future studies are required to further elucidate the effect of $\mathrm{CPB}$, cardioplegia, and reperfusion on NOS activity regulation and ROS formation, as well as their effects on tissues.

In conclusion, the data of the present study for the first time provide direct evidence for ROS-induced myocardial injury after ischemia and reperfusion in patients subjected to cardioplegia and CPB. These findings suggest a rational basis for investigation of ROS scavengers, selective NOS inhibition, or both as adjuncts to reperfusion strategies. Specifically, direct myocardial nitrotyrosine and 8-isoprostane detection in the experimental setting might be used to provide a rational basis for dose selection before clinical evaluation of antioxidant reperfusion strategies.

We thank Martin Hellmich, PhD, Department for Medical Statistics, Informatics, and Epidemiology, University of Cologne for statistical advice.

\section{References}

1. Reilly MP, Delanty N, Roy L, Rokach J, Callaghan PO, Crean P, et al. Increased formation of the isoprostanes IPF2alpha-I and 8-epi-prostaglandin F2alpha in acute coronary angioplasty: evidence for oxidant stress during coronary reperfusion in humans. Circulation. 1997;96: 3314-20.

2. Sekili S, McCay PB, Li X-Y, Zughaib M, Sun J-Z, Tang L, et al. Direct evidence that the hydroxyl radical plays a pathogenetic role in myocardial 'stunning' in the conscious dog and demonstration that stunning can be markedly attenuated without subsequent adverse effects. Circ Res. 1993;73:705-23.

3. Li X-Y, McCay PB, Zughaib M, Jeroudi MO, Triana F, Bolli R. Demonstration of free radical generation in the 'stunned' myocardium in the conscious dog and identification of the major differences between conscious and open-chest dogs. J Clin Invest. 1993;92:1025-41.

4. Holley AK, Cheeseman KH. Measuring free radical reactions in vivo. Br Med Bull. 1992;49:494-505.

5. Davies SW, Ranjadayalan K, Wickens DC, Dorinandy TL, Tinunis AD. Lipid peroxidation associated with successful thrombolysis. Lancet. 1990;335:741-3.

6. Grech ED, Dodd HF, Bellamy CM, Perry RA, Morrison WL, Ramsdale DR. Free radical generation during angioplasty reperfusion for acute myocardial infarction. Lancet. 1993;341:990-1.

7. Coghlan JG, Flitter WD, Clutton SM, Panda R, Daly R, Wright G, et al. Allopurinol pretreatment improves postoperative recovery and reduces lipid peroxidation in patients undergoing coronary bypass grafting. J Thorac Cardiovasc Surg. 1994;107:248-56.

8. Basu S, Nozari A, Liu XL, Rubertsson S, Wiklund L. Development of a novel biomarker of free radical damage in reperfusion injury after cardiac arrest. FEBS Lett. 2000;470:1-6.

9. Mihm MJ, Coyle CM, Schanbacher BL, Weinstein DM, Bauer JA. Peroxynitrite induced nitration and inactivation of myofibrillar creatine kinase in experimental heart failure. Cardiovasc Res. 2001;49:798807.

10. Cesselli D, Jakoniuk I, Barlucchi L, Beltrami AP, Hintze TH, NadalGinard B, et al. Oxidative stress-mediated cardiac cell death is a major determinant of ventricular dysfunction and failure in dog dilated cardiomyopathy. Circ Res. 2001;89:279-86.

11. Waugh RJ, Murphy RC. Mass spectrometric analysis of four regioisomers of $\mathrm{F}_{2}$ isoprostanes formed by free radical oxidation of arachidonic acid. J Am Soc Mass Spectrom. 1996;7:490-9.
12. Mehlhorn U, Bloch W, Krahwinkel A, LaRose K, Geissler HJ, Hekmat $\mathrm{K}$, et al. Activation of myocardial constitutive nitric oxide synthase during coronary artery surgery. Eur J Cardiothorac Surg. 2000; 17:305-11.

13. Hayashi Y, Sawa Y, Ohtake S, Fukuyama N, Nakazawa H, Matsuda $\mathrm{H}$. Peroxynitrite formation from human myocardium after ischemiareperfusion during open heart operation. Ann Thorac Surg. 2001;72: 571-6.

14. Clements FM, Harpole DH, Quill T, Jones RH, McCann RL. Estimation of left ventricular volume and ejection fraction by two-dimensional transesophageal echocardiography: comparison of short axis imaging and simultaneous radionuclide angiography. $\mathrm{Br} J$ Anesth. 1990;64:331-6.

15. De Vente J, Steinbusch HWM. On the stimulation of soluble and particulate guanylate cyclase in the rat brain and the involvement of nitric oxide as studied by cGMP immunocytochemistry. Acta Histochem. 1992;92:13-38.

16. Nonami Y. The role of nitric oxide in cardiac ischemia-reperfusion injury. Jpn Circ J. 1997;61:119-32.

17. Lefer DJ, Nakanishi K, Johnson WE, Vinten-Johansen J. Antineutrophil and myocardial protection actions of a novel nitric oxide donor after acute myocardial ischemia and reperfusion in dogs. Circulation. 1993:88:2337-50.

18. Welsh N, Eizirik DL, Bendtzen K, Sandler S. Interleukin-1 betainduced nitric oxide production in isolated rat pancreatic islets requires gene transcription and may lead to inhibition of the Krebs cycle enzyme aconitase. Endocrinology. 1991;129:3167-73.

19. Henry Y, Lepoivre M, Drapier JC, Ducrocq C, Boucher JL, Guissani A. EPR characterization of molecular targets for NO in mammalian cells and organelles. FASEB J. 1993;7:1124-34.

20. Lepoivre M, Flaman JM, Henry Y. Early loss of the tyrosyl radical in ribonucleotide reductase of adenocarcinoma cells producing nitric oxide. J Biol Chem. 1992;267:22994-3000.

21. Ferdinandy P, Danial H, Ambrus I, Rothery RA, Schulz R. Peroxynitrite is a major contributor to cytokine-induced myocardial contractile failure. Circ Res. 2000;87:241-7.

22. Bloch W, Mehlhorn U, Krahwinkel A, Reiner M, Dittrich M, Schmidt A, et al. Ischemia increases detectable endothelial nitric oxide synthase in rat and human myocardium. Nitric Oxide. 2001; 5:317-33.

23. Depré C, Fierain L, Hue L. Activation of nitric oxide synthase by ischaemia in the perfused heart. Cardiovasc Res. 1997;33:82-7.

24. Kijima Y, Hashimura K, Matsu-ura Y, Kato Y, Yasuda T, Ueda T, et al. Transcardiac 8-iso-prostaglandin $\mathrm{F}$ (2alpha)generation from acute myocardial infarction heart: insight into abrupt reperfusion and oxidant stress. Prostaglandins Leukot Essent Fatty Acids. 2001;64:161-6.

25. Brahmajothi MV, Campbell DL. Heterogeneous basal expression of nitric oxide synthase and superoxide dismutase isoforms in mammalian heart. Implications for mechanisms governing indirect and direct nitric oxide-related effects. Circ Res. 1999;85:575-87.

26. Digerness SB, Harris KD, Kirklin JW, Urthaler F, Viera L, Beckman JS, et al. Peroxynitrite irreversibly decreases diastolic and systolic function in cardiac muscle. Free Radic Biol Med. 1999; 27:1386-92.

27. Feng Q, Lu X Jones DL, Shen J, Arnold JM. Increased inducible nitric oxide synthase expression contributes to myocardial dysfunction and higher mortality after myocardial infarction in mice. Circulation. 2001;104:700-4.

28. Gupte SA, Okada T. Prostaglandins and nitric oxide mediate superoxide-induced myocardial contractile dysfunction in isolated rat hearts. J Mol Cell Cardiol. 2001;33:1107-17.

29. Mobert J, Becker BF, Zahler S, Gerlach E. Hemodynamic effects of isoprostanes (8-iso-prostaglandin F2alpha and E2) in isolated guinea pig hearts. J Cardiovasc Pharmacol. 1997;29:789-94.

30. Gupte SA, Okada T, Tateyama M, Ochi R. Activation of TxA2/ $\mathrm{PGH} 2$ receptors and protein kinase $\mathrm{C}$ contribute to coronary dysfunction in superoxide treated rat hearts. $\mathrm{J} \mathrm{Mol} \mathrm{Cell} \mathrm{Cardiol.}$ 2000;32:937-46. 